\title{
Wave function of the Universe and CMB fluctuations
}

\author{
S. P. de Alwis* \\ Physics Department, University of Colorado, Boulder, Colorado 80309, USA
}

(Received 18 April 2019; published 30 August 2019)

\begin{abstract}
The Hartle-Hawking and tunneling (Vilenkin) wave functions are treated in the Hamiltonian formalism. We find that the leading (i.e., quadratic) terms in the fluctuations around a maximally symmetric background are indeed Gaussian (rather than inverse Gaussian), for both types of wave function, when properly interpreted. However, the suppression of non-Gaussianities-and hence the recovery of the Bunch-Davies state-is not transparent.
\end{abstract}

DOI: 10.1103/PhysRevD.100.043544

\section{INTRODUCTION}

Inflationary cosmology is not past eternal. Under the assumption that the average Hubble parameter $\langle H\rangle>0$ i.e., that on average the Universe has been expanding in the past—both null and timelike geodesics cannot be arbitrarily extended to the past [1]. In fact unlike the cosmological singularity theorems, which relied on the use of the weak energy condition (violated by inflationary cosmology), this argument does not need such a condition.

On the other hand the standard calculation of the scalar and tensor fluctuation spectrum around the inflationary background assumes that in the distant past [conformal time $\eta \rightarrow-\infty(1-i \epsilon)]$, the inflationary background remains valid, and correlation functions in any state of the system (defined in the interaction picture) at some conformal time $\tau$ are related to those in the "Bunch-Davies" (BD) vacuum state $\mid 0>$ by the "in-in" formula $[2,3]$,

$$
\begin{array}{r}
\frac{\langle\Omega(\tau)|\hat{W}(\tau)| \Omega(\tau)\rangle}{\langle\Omega(\tau) \mid \Omega(\tau)\rangle}=\left\langle 0\left|U^{\dagger}(\tau,-\infty) W(\tau) U(\tau,-\infty)\right| 0\right\rangle, \\
U(\tau,-\infty)=T \exp \left(-\frac{i}{\hbar} \int_{-\infty(1-i \epsilon)}^{\tau} \hat{H}_{1 I}\left(\tau^{\prime}\right) d \tau^{\prime}\right) .
\end{array}
$$

In the above the Hamiltonian for fluctuations around the (time-dependent) inflationary background is taken to be of the form $H_{0}(t)+H_{1}(t)$, where the first term governs quadratic fluctuations and the second cubic and higher order fluctuations. Also $\hat{H}_{1 I}$ is $H_{1}$ in the interaction picture.

\footnotetext{
*dealwiss@colorado.edu
}

Published by the American Physical Society under the terms of the Creative Commons Attribution 4.0 International license. Further distribution of this work must maintain attribution to the author(s) and the published article's title, journal citation, and DOI. Funded by SCOAP ${ }^{3}$.
The important point to note here is that both $H_{0}$ and $H_{1}$ are time dependent, with this dependence given by the slow roll inflationary background metric. The projection onto the matrix element in the $\mathrm{BD}$ vacuum is a consequence of taking the limit of infinite negative conformal time. This however seems to be in apparent conflict with the theorem of Ref. [1], in which inflation is not past eternal.

This motivates us to search for some explanation as to the emergence of an inflationary background starting from "nothing." This has been discussed since the early 1980s, and there are two main proposals. One is the "no boundary" wave function of Hartle and Hawking ( $\mathrm{HH})$ [4]. The other is the tunneling $(\mathrm{T})$ wave function of Vilenkin [5]. ${ }^{1}$ While both wave functions could in principle be valid solutions to the Wheeler-DeWitt equation without truncation-in practice the explicit forms of the wave functions have been obtained only in the so-called minisuperspace model, where only the temporal dependence of the fields is kept.

Recently this basis for justifying the BD state in the inflationary background has been questioned [9-12]. Using the Picard-Lefschetz theory of saddle point approximations to the integral over the lapse $N$ of the Arnowitt-DeserMisner formulation of general relativity, it was claimed in Ref. [9] that this method justifies the tunneling wave function but not the $\mathrm{HH}$ wave function. In a subsequent paper [10] Feldbrugge et al. then argued that quadratic scalar/tensor fluctuations around both $\mathrm{T}$ and $\mathrm{HH}$ wave functions were unsuppressed, having an inverse Gaussian form. This seemed to cast doubt on the quantum cosmology justification for a smooth BD beginning to inflationary cosmology.

\footnotetext{
${ }^{1}$ For a recent discussion and references to the early literature, see Ref. [6]. That paper also addresses issues similar to those in this paper, but only for the $\mathrm{T}$ wave function and from a different perspective. See also Ref. [7] and references therein for a related discussion, as well as Ref. [8] for a loop quantum gravity perspective.
} 
In a rebuttal of these claims one of the original proponents of the HH wave function (Hartle) and collaborators [13], argued that there is a choice of contour that justifies the $\mathrm{HH}$ wave function. Furthermore, whereas the $\mathrm{T}$ wave function (following from the contour of Ref. [9]) was indeed unstable due to unsuppressed fluctuations (as argued a while ago also in the references quoted in Ref. [13]), the HH wave function is not. Subsequently in Ref. [11] it was argued that the contour chosen in Ref. [13] actually should pick up subdominant saddle points, which restores the unsuppressed fluctuations in the $\mathrm{HH}$ wave function as well.

A new round of claims and counterclaims were made in 2018. Reference [14] considered a generalization of minisuperspace, replacing the round $S_{3}$ by axial Bianchi IX geometry. Diaz Dorronsoro et al. argued, using a circular contour for the integration over the lapse $N$, that the $\mathrm{HH}$ wave function is well defined, and hence that the original $\mathrm{HH}$ wave function was stable under deformations. This choice of contour was criticized in Ref. [12] on the grounds that it was not physically well motivated and that it leads to "mathematical and physical inconsistencies." Finally in a very recent paper Vilenkin and Yamada [6] argued, provided that certain boundary/initial conditions on the scalar fluctuations are satisfied, that the scalar field fluctuations around the tunneling wave function $(\mathrm{T})$ are well behaved.

Our point of view is that quantum gravity should be defined in terms of the Wheeler-DeWitt (WdW) equation, which is a constraint equation whose solutions are possible wave functions of the Universe. The functional integral definition of the wave function is simply a method for computing it. ${ }^{2}$ But there are equally valid approaches to solving the WdW equation, such as the WKB method. In this paper we will use the latter method to discuss the possible solutions to the WdW equation in the semiclassical approximation, and to rederive solutions that had been obtained before using the functional integral method. Including the fluctuations around the cosmological de Sitter background, we find four independent solutions. Different choices of contour on the two sides of this dispute just correspond to different choices of integration constants. We do not believe that there is any "fundamental principle" that dictates one or other choice, as seems to be the position of Feldbrugge et al. [9]. In fact as the authors point out, their contour leads to unsuppressed-i.e., inverse Gaussian -fluctuations, in contradiction to what has been observed. On the other hand as shown in Refs. [13,14], there exists an alternate choice of contour which leads to the $\mathrm{HH}$ wave function with suppressed fluctuations.

\footnotetext{
${ }^{2}$ This appears to be the position of Hartle, one of the founding fathers of quantum cosmology, and his collaborators as well-see for instance the recent paper [15].
}

This paper is aimed at reconciling these different points of view. Our main argument is that the WdW equation implies that there is no notion of time (and therefore of time ordering). It is essentially like the time-independent Schrödinger equation whose solutions are stationary states. This of course is the famous "problem of time" in quantum gravity. One can compare the probabilites for different configurations of the fields of the system and its geometry. But there is no notion of which configuration is prior to which.

Thus in the present context there are four different particular solutions of the $\mathrm{WdW}$ equations in the semiclassical approximation, both inside and outside the effective potential barrier, which can then be matched as in the standard WKB procedure. By appropriate choices of integration constants, these solutions can be organized either into tunneling-type wave functions or into HH-type (real) wave functions, both with suppressed Gaussian fluctuations. On the other hand one also has (for both cases) solutions with unsuppressed (inverse Gaussian) fluctuations. These are the solutions that were obtained by Feldbrugge et al. $[10,11]$ from their integration contour for the lapse. Clearly these are unphysical (certainly inconsistent with the observed smooth homogeneous isotropic Universe) and hence should be rejected.

We will first discuss two simple problems which illustrate one of the main points which we wish to make-namely, that there is no advantage to using the functional integral and the Picard-Lefschetz method to solve the WdW equation wherever it is possible to solve the equation directly in the semiclassical approximation, which is the case in all of these examples. In fact in the simplest example, that of oneparticle nonrelativistec quantum mechanics, derived from a time-reparametrization invariant action leading to a $\mathrm{WdW}$ equation, it is clear that while one can indeed define the semiclassical solution in terms of an integral over the lapse - the dependence on the latter drops out of the classical action when one uses the constraint equation. Next we discuss the Schwinger process, where essentially the same is true. In effect we argue that one needs some physical input to decide what particular solution to pick, and that one cannot do this on mathematical grounds or on the basis of an a priori notion of causality. We then review the minisuperspace solution for Universe creation from nothing - both the $\mathrm{HH}$ and the $\mathrm{T}$ cases. Finally we discuss fluctuations around minisuperspace. We will find that, properly interpreted, both the $\mathrm{HH}$ and $\mathrm{T}$ cases lead to suppressed Gaussian fluctuations for the wave function in the classical regime, contrary to the claims in Refs. [10-12]. On the other hand this wave function (in both the $\mathrm{HH}$ or $\mathrm{T}$ cases) necessarily has non-Gaussian (i.e., cubic and higher powers of fluctuations) terms. In other words the emergence of the BunchDavies vacuum wave function, which is just Gaussian, does not appear to have any explanation from these considerations. 


\section{QUANTUM MECHANICS EXAMPLES}

In the following we will discuss two examples which show that both the Hamiltonian argument and the path integral one agree, and that the latter does not resolve the ambiguity involved in the choice of wave function.

\section{A. Particle in a potential}

We consider a one-dimensional nonrelativistic particle action with time-reparametrization invariance:

$$
\begin{gathered}
S=\int_{0}^{1} d t\left[N(t)^{-1} \frac{1}{2} \dot{x}^{2}-N(t)(V-E)\right], \\
p_{x}=\frac{\dot{x}}{N}, \quad p_{N}=0, \\
\mathcal{H}=p_{x} \dot{x}-L=N\left(\frac{1}{2} p^{2}+V-E\right) .
\end{gathered}
$$

We have the secondary constraint

$$
\dot{p}_{N}=\left\{p_{N}, \mathcal{H}\right\}=\left(\frac{1}{2} p_{x}^{2}+V-E\right)=0
$$

i.e., the Hamiltonian is weakly zero,

$$
\mathcal{H} \approx 0 .
$$

This is of course completely equivalent to the usual energy conservation equation. Also we have

$$
\dot{N}=\{N, \mathcal{H}\}=0 .
$$

So we can choose the gauge $N=$ constant. The (timeindependent) Schrödinger equation is then the same as the Wheeler-DeWitt equation, i.e., with $p_{x} \rightarrow \hat{p}_{x}=-i \hbar \frac{d}{d x}$,

$$
\hat{H} \Psi(x)=\left(-\frac{\hbar^{2}}{2} \frac{d^{2}}{d x^{2}}+V(x)-E\right) \Psi(x)=0 .
$$

Solving this by putting $\Psi[q, \phi]=e^{\frac{i}{\hbar} S[q, \phi]}$ with $S=S_{0}+$ $\hbar S_{1}$ gives

$$
\frac{1}{2}\left(\frac{d S_{0}}{d x}\right)^{2}+V-E=0, \quad \frac{d S_{1}}{d x}=\frac{i}{2} \frac{d}{d x} \ln \frac{d S_{0}}{d x} .
$$

This gives, in the semiclassical approximation, the usual WKB result

$$
\Psi(x)=\frac{c}{|2(E-V(x))|^{1 / 4}} e^{ \pm \frac{i}{\hbar} \int^{x} \sqrt{2\left(E-V\left(x^{\prime}\right)\right)} d x^{\prime}},
$$

and clearly both solutions are allowed. In the regime where $V(x)>E$, we have both an exponentially rising and a falling solution with absolutely no mathematical reason for discarding one or the other. Similarly (assuming that the potential is greater than zero only over a finite interval so that what we have is a barrier rather than an infinite wall) one has outgoing and incoming solutions, again with no reason to discard either.

Now consider the path integral for the Feynman kernel for this problem. The Feynman kernel to go from $x_{0}$ at time $t_{0}=0$ to $x_{1}$ at $t_{1}=1$ is (note $K$ is a function of the first four variables and a functional of $N$ )

$$
\begin{aligned}
K & \left(x_{1}, t_{1} ; x_{0}, t_{0}: N\right] \\
= & \left\langle x_{1}\left|T e^{-\frac{i}{h} \int_{t_{0}}^{t_{1}} \hat{\mathcal{H}} d t}\right| x_{0}\right\rangle \\
= & \int \prod_{t_{0}}^{t_{1}} d p\left(t_{i}\right) \\
& \times \int \prod_{x\left(t_{0}\right)=x_{0}}^{x\left(t_{1}\right)=x_{1}} d x\left(t_{i}\right) e^{\frac{i}{\hbar} \int_{t_{0}}^{t_{1}} d t\left[p(t) \dot{x}(t)-N(t)\left(\frac{1}{2} p^{2}(t)+V(x(t))-E\right)\right]} .
\end{aligned}
$$

Let us first integrate over $p$ to get the Lagrangian form of the path integral

$$
K\left(x_{1} ; x_{0}\right)=\int \frac{[d N]}{\sqrt{N}}[d x] e^{\frac{i}{h} \int_{0}^{1} d t\left[N(t)^{-1} \frac{1}{2} \dot{x}^{2}-N(t)(V-E)\right]} .
$$

From Eq. (3) we have the following equations of motion:

$$
\begin{gathered}
\delta_{N} S=0 \Rightarrow \frac{\dot{x}^{2}}{2}+N^{2} V=N^{2} E, \\
\delta_{x} S=0 \Rightarrow \ddot{x}=-N^{2} V^{\prime}(x) .
\end{gathered}
$$

Let us specialize to a linear potential [as in the minisuperspace case with a positive cosmological constant (CC)] $V=V_{0}-\Lambda x, \Lambda>0$. The equation for $x$ is solved by

$$
x=\frac{1}{2} N^{2} \Lambda t^{2}+\left(x_{1}-x_{0}-\frac{1}{2} N^{2} \Lambda\right) t+x_{0} .
$$

Evaluating Eq. (10) at $t=0$ and using Eq. (12) gives a quartic equation for $N$ (after gauge fixing $\dot{N}=0$ )

$\frac{\Lambda^{2}}{8} N^{4}-N^{2}\left(E-V_{0}+\frac{1}{2} \Lambda\left(x_{1}+x_{0}\right)\right)+\frac{1}{2}\left(x_{1}-x_{0}\right)^{2}=0$,

which has the four solutions

$$
N= \pm \frac{\sqrt{2}}{\Lambda}\left[\left(E-V_{0}+\Lambda x_{1}\right)^{1 / 2} \pm\left(E-V_{0}+\Lambda x_{0}\right)^{1 / 2}\right],
$$

corresponding to the four (in general complex) saddle points found in Ref. [9] for the minisuperspace case. 
In effect Feldbrugge et al. would have calculated Eq. (9) in the saddle point approximation and decided which contour to integrate $N$ over, and hence which saddle points to pick up based on a Picard-Lefschetz analysis. In fact it has been argued by those authors that one should integrate only over positive $N$.

However, the classical action is completely independent of these. Solving Eq. (10) for $\dot{x}$ and substituting in Eq. (3),

$$
\begin{aligned}
S & =\int_{0}^{1} d t\left[N^{-1} N^{2}(E-V)+N(E-V)\right]=\int_{0}^{1} d t[2 N(E-V)] \\
& =\int_{x_{0}}^{x_{1}} \frac{d x}{\dot{x}} 2 N(E-V)= \pm \int_{x_{0}}^{x_{1}} \frac{d x}{N \sqrt{2(E-V)}} N 2(E-V) \\
& = \pm \int_{x_{0}}^{x_{1}} d x \sqrt{2(E-V)} .
\end{aligned}
$$

This is independent of the saddle points for $N$ and gives for $K\left(x_{1} ; x_{0}\right)$ as expected two different results, as was the case with the wave function calculation (8).

In any case as we argued in the Introduction, the analog of the Feynman kernel is not a physically interesting quantity to calculate. The probability for tunneling is given by the squares of (or ratios of squares of) wave functions. Again the point is that we are looking at energy eigenstates and asking what the probability of finding the position of the particle (which of course is not a variable which commutes with the Hamiltonian) on one or the other side of the potential barrier.

\section{B. Particle creation in an electric field ( $E$ field)}

In Ref. [16] Brown and Teitelboim used a Euclidean instanton to describe pair creation in an electric field (the Schwinger process) in $1+1$ dimensions, then extended it to brane nucleation in higher dimensions. We will focus just on the former in flat space and will ignore the dynamics of the electromagnetic field:

$$
S=-m \int d s\left(-\eta_{\mu \nu} \dot{x}^{\mu} \dot{x}^{\nu}\right)^{1 / 2}-e \int d s \dot{x}^{\mu} A_{\mu}, \quad \dot{x}^{\mu} \equiv \frac{d x^{\mu}}{d s} .
$$

Introduce a proper time metric factor $N(s)$ to write the action in quadratic form, and choose the gauge potential for a constant electric field $E$ as $A_{\mu}=(E x, 0)$, so

$$
S=-\int d s\left\{\frac{m}{2 N(s)}\left(\dot{t}^{2}-\dot{x}^{2}\right)+\frac{m}{2} N(s)\right\}-e E \int d s \dot{t} x .
$$

In the canonical formalism we have conjugate momenta

$$
\pi_{t}=-\frac{m}{N} \dot{t}-e E x, \quad \pi_{x}=m \frac{\dot{x}}{N}, \quad \pi_{N}=0,
$$

and Hamiltonian

$\mathcal{H}=N\left(-\frac{\pi_{t}^{2}}{2 m}+\frac{\pi_{x}^{2}}{2 m}-\frac{(e E x)^{2}}{2 m}-\frac{e}{m} \pi_{t} E x+\frac{m}{2}\right) \equiv N H$.

The dynamics is given by Hamilton's equations for the phase space variables $\{\alpha\}=N, \pi_{N}, x, \pi_{x}, t, \pi_{t}$,

$$
\dot{\alpha}=\{\alpha, \mathcal{H}\}
$$

with the following relations/constraints.

Poisson brackets:

$$
\left\{N, \pi_{N}\right\}=\left\{x, \pi_{x}\right\}=\left\{t, \pi_{t}\right\}=1
$$

Primary constraints are

$$
\pi_{N} \approx 0
$$

Secondary constraints are

$$
\dot{\pi}_{N}=\left\{\pi_{N}, \mathcal{H}\right\}=-H \approx 0 .
$$

The equations of motion are

$\dot{N}=\{N, \mathcal{H}\}=0, \quad \dot{t}=-\frac{N}{m}\left(\pi_{t}+e E x\right), \quad \dot{x}=N \frac{\pi_{x}}{m}$,

$\dot{\pi}_{t}=0, \quad \dot{\pi}_{x}=N(e E)^{2} \frac{x}{m}$.

Since $\pi_{t}$ is constant, let us choose

$$
\pi_{t}=\pi_{t 0}=0
$$

Also in the passage to quantum mechanics (QM), $\pi_{q} \rightarrow-i \hbar \frac{\delta}{\delta q}$, etc., acting on the Schrödinger wave function $\Psi$. In particular $\pi_{N} \approx 0$ implies

$$
\frac{\delta}{\delta N} \Psi=0
$$

and $H \approx 0$ implies the "Wheeler-DeWitt" equation

$$
H\left(q,-i \hbar \frac{\delta}{\delta q}\right) \Psi(q)=0
$$

(We are assuming there is no boundary in space and hence no boundary Hamiltonian.)

In the WKB approximation (ignoring the prefactor)

$$
\Psi \propto e^{\frac{i}{\hbar} S_{c l}}
$$

where the classical action (evaluated on a solution) is 


$$
S_{c l}=\int d s\left[\pi_{t} \dot{t}+\pi_{x} \dot{x}+\pi_{N} \dot{N}-\mathcal{H}\right)=\int \pi_{x} d x
$$

In the last step we used Eqs. (18) and (21). Again, as in the previous example, we see that imposing the classical constraints on the motion leaves the classical action completely independent of the lapse $N$. Also from Eqs. (19) and (21) we have, solving for $\pi_{x}$,

$$
\pi_{x}= \pm|e E| \sqrt{x^{2}-\left(\frac{m}{e E}\right)^{2}} .
$$

Evaluating the integral between the two turning points, one gets (defining $\gamma=m /|e E|$ )

$$
\begin{aligned}
S_{c l} & = \pm|e E| \int_{-\gamma}^{+\gamma} d x \sqrt{x^{2}-\gamma^{2}} \\
& = \pm|e E|\left[\frac{1}{2} x \sqrt{x^{2}-\gamma^{2}}-\frac{1}{2} \gamma^{2} \ln \left(x+\sqrt{x^{2}-\gamma^{2}}\right]_{-\gamma}^{+\gamma}\right. \\
& = \pm \frac{i \pi m^{2}}{2|e E|}(2 n+1), \quad n \in \mathcal{Z}^{\geq} .
\end{aligned}
$$

This gives a probability (from the dominant solution $n=0$ )

$$
P \propto|\Psi|^{2} \propto e^{-\frac{\pi m^{2}}{|e E|}}
$$

which is in agreement with Brown and Teitelboim's instanton calculation (and the leading term in Schwinger's calculation). Note that here we have rejected the possible positive sign in the exponent on physical grounds since otherwise we would have $P$ rising with a decreasing electric field $E$. In other words there is no way that one can get the right sign from the formalism.

Let us redo the calculation in the Lagrangian formulation with the lapse gauge fixed to $N(s)=N$ (a constant as in Ref. [9]). The action is again given by Eq. (14), and the Lagrangian equations of motion are

$$
\ddot{t}=\alpha \dot{x}, \quad \ddot{x}=\alpha \dot{t}, \quad \alpha \equiv-\frac{e E N}{m},
$$

from varying with respect to $x^{\mu}$, and from varying with respect to $N$,

$$
-\frac{m}{2 N^{2}}\left(\dot{t}^{2}-\dot{x}^{2}\right)+\frac{m}{2}=0
$$

Define $x^{ \pm}=t \pm x$ so that the above equations become

$$
\ddot{x}^{ \pm}= \pm \alpha \dot{x}^{ \pm}, \quad N^{2}=\dot{x}^{+} \dot{x}^{-} .
$$

With an appropriate choice of initial conditions we have the solutions

$$
\dot{x}^{ \pm}=\dot{x}_{0}^{ \pm} e^{ \pm \alpha s}, \quad x^{ \pm}= \pm \frac{\dot{x}_{0}^{ \pm}}{\alpha} e^{ \pm \alpha s}, \quad N^{2}=\dot{x}_{0}^{+} \dot{x}_{0}^{-} .
$$

The equation for the orbit is

$$
t^{2}-x^{2}=-\frac{\dot{x}_{0}^{+} \dot{x}_{0}^{-}}{\alpha^{2}}=-\frac{m^{2}}{(e E)^{2}},
$$

where in the last step we used the last equation of Eq. (26). Note that at $t=0$ we have

$$
x(t=0)= \pm \frac{m}{e E} \equiv \pm \gamma .
$$

In the Brown-Teitelboim description of pair creation, the particle on the left propagates backward in time (antiparticle) $t$, while the particle on the right propagates forward. This is to be interpreted as pair creation at time $t=0$ at the points given by Eq. (28). Along the spacelike tunneling trajectory $\dot{x}_{0}^{+} \dot{x}_{0}^{-}=-\left|\dot{x}_{0}^{+} \dot{x}_{0}^{-}\right|$, which implies that the saddle points for the integration over $N$ are pure imaginary, $N= \pm i \sqrt{\left|\dot{x}_{0}^{+} \dot{x}_{0}^{-}\right|}$. But the point is that the $E$ dependence of the action at the classical solutions comes only from the second term of Eq. (14), which is independent of which solution for $N$ one chooses. Evaluating the last term in the action over the tunneling trajectory gives an imaginary part to the action from the term,

$$
e E \int_{x=-\gamma}^{x=\gamma} d s \dot{t} x=-\int_{-\gamma}^{\gamma} d x t(x)=\mp \int_{-\gamma}^{\gamma} d x \sqrt{x^{2}-\gamma^{2}}
$$

which is the same as before, i.e., Eq. (24), and hence gives the same probability for pair creation, Eq. (25).

The point of this exercise was to show that picking one or other solution for $N$ does not resolve the sign ambiguity that we had in the Hamiltonian discussion, as there this ambiguity comes from the two solutions for $t$, which in that case was from solving the Hamiltonian constraint, while here it comes from solving the orbit equation (27).

Feldbrugge et al. [10] claim to be able to resolve this difference by computing the Feynman kernel for propagation from $t_{0}, x_{0}$ to $t_{1}, x_{1}$ (as in the case of the simple QM calculation of the previous example). However, the reason for this resolution is the imposition of a certain "causality" criterion on the integral over $N$. However, the forward direction of time is ambiguous in this one-particle quantum mechanics discussion of what is essentially a field theoretic process. Does one regard this as an electron traveling first backward in time and then forward or a positron traveling forward in time and then backward or vice versa? In the field theoretic argument on the other hand unitarity can resolve this ambiguity as in the original Schwinger calculation. 


\section{MINISUPERSPACE}

\section{A. Background wave function}

Let us now discuss minisuperspace in the Hamiltonian formalism. The action is given by (after setting $\left.M_{P}^{-1}=8 \pi G=1\right)$

$$
S=\int_{0}^{1} d t\left(-N^{-1} 3 a \dot{a}^{2}+\left(3 k a-a^{3} \Lambda\right) N\right) .
$$

Note for future reference that the actual action (in $3+1$ dimensions) has a factor of the unit three sphere volume and is $2 \pi^{2} S$. Also $\dot{x} \equiv \frac{d x}{d t}$. Change the variable to $q=a^{2}$, $N \rightarrow N / a$ :

$$
\begin{gathered}
S=\int d t\left(-\frac{3}{4 N} \dot{q}^{2}+N(3 k-\Lambda q)\right) \\
\pi_{N}=0, \quad \pi_{q}=-\frac{3}{2 N} \dot{q}, \quad \dot{q}=-\frac{2 N \pi_{q}}{3}, \\
\mathcal{H}=N\left(-\frac{\pi_{q}^{2}}{3}+(\Lambda q-3 k)\right) \equiv N H .
\end{gathered}
$$

The primary and secondary constraints are

$$
\pi_{N}=0, \quad \dot{\pi}_{N}=\left\{\pi_{N}, \mathcal{H}\right\}=H \approx 0 .
$$

So on a classical trajectory

$$
\pi_{q}^{2}=3(\Lambda q-3 k), \quad \pi_{q}= \pm 3 \sqrt{\frac{\Lambda}{3} q-k} .
$$

The Wheeler-DeWitt equation for the system is obtained by putting $\pi_{q} \rightarrow-i \hbar \partial / \partial q$ in the Hamiltonian constraint and is

$$
\left\{+\frac{\hbar^{2}}{3} \frac{\partial^{2}}{\partial q^{2}}+(\Lambda q-3)\right\} \Psi=0
$$

Consider tunneling to a de Sitter space with $\Lambda>0, k=1$. The classical action is

$$
\begin{aligned}
\int_{q_{0}}^{q_{1}} \pi_{q} d q & = \pm 3 \int_{q_{0}}^{q_{1}} d q \sqrt{\frac{\Lambda}{3} q-k} \\
& =\mp \frac{6 i}{\Lambda}\left[\left(1-\frac{\Lambda}{3} q_{1}\right)^{3 / 2}-\left(1-\frac{\Lambda}{3} q_{0}\right)^{3 / 2}\right] .
\end{aligned}
$$

This is pure imaginary for "under the barrier" propagation $q_{0}, q_{1}<\frac{3}{\Lambda}$. In the semiclassical approximation (and ignoring the fluctuations) this gives the transition amplitude (ignoring prefactors)

$$
\begin{aligned}
& K\left(a_{1} ; a_{0}\right) \\
& \sim \exp \left[i 2 \pi^{2} \int_{q_{0}}^{q_{1}} \pi_{q} d q\right] \\
& =\exp \left[ \pm \frac{12 \pi^{2}}{\Lambda}\left\{\left(1-\frac{\Lambda}{3} a_{1}^{2}\right)^{3 / 2}-\left(1-\frac{\Lambda}{3} a_{0}^{2}\right)^{3 / 2}\right\}\right]
\end{aligned}
$$

Thus again the Hamiltonian analysis shows that both signs are allowed; i.e., both $\mathrm{HH}$ and $\mathrm{T}$ are valid solutions. The two signs come again from the fact that the $H$ constraint is quadratic in $\pi$. As in the case of the particle in an E field, the sign has to be chosen on physical grounds. One might argue as in the latter case that the upper sign (T) is physically more plausible. Actually the probability of finding a scale factor $a_{1}$,

$$
P\left(a_{1}\right) \sim\left|\Psi\left(a_{1}\right)\right|^{2},
$$

depends on the initial wave function since

$$
\Psi\left(a_{1}\right)=\int d a_{0} \mu\left(a_{0)} K\left(a_{1} ; a_{0}\right) \Psi_{0}\left[a_{0}\right] .\right.
$$

If we take the "initial" minisuperspace Universe to be an eigenstate of the scale factor with zero scale factor (nothing), $\Psi_{0} \propto \delta\left(a_{0}\right)$. So

$$
P\left(a_{1}\right) \sim \exp \left[\mp \frac{24 \pi^{2}}{\Lambda}\left\{1-\left(1-\frac{\Lambda}{3} a_{1}^{2}\right)^{3 / 2}\right\}\right] .
$$

The upper sign gives a falling probability with increasing $a_{1}$ [T (Vilenkin)] while the lower sign a rising probability $(\mathrm{HH})$.

We should note in passing that this wave function is an asymptotic expression that is valid only in the region that is not only inside but also far from the turning point. In particular it cannot be used at the turning point $a=\sqrt{\frac{3}{\Lambda}}$ itself. We shall discuss this further in the next subsection.

Feldbrugge et al. [9] claim to fix the sign ambiguity by first integrating over $q$ and then doing an integral over positive $N$ and picking what they claim is the correct saddle point. Somehow this seems to imply that the above calculation must fail for the "wrong" sign. As in the case of the nonrelativistic particle and that of the charged particle in an E field, it is clear from this analysis that this ambiguity cannot be fixed by an extraneous notion of causality.

\section{B. Hartle-Hawking or Tunneling}

We argued in the previous subsections that the choice of the overall sign of the exponent in Eqs. (33) and (35) is not determined by any mathematical consistency argument, but it may be fixed by physical considerations. To discuss this, we need to match each under the barrier (real) wave function to the appropriate (linear combination of) oscillating 
wave functions. This is done by the standard WKB matching conditions.

In fact if one ignores the fluctuations, one simply has a linear potential, and the exact solution is well known (see, e.g., Ref. [17]). Thus defining

$$
z=\left(\frac{3}{\hbar^{2} \Lambda^{2}}\right)^{1 / 3}(3-\Lambda q)
$$

the Wheeler-DeWitt equation (32) becomes the Airy function equation

$$
\frac{d^{2} f(z)}{d z^{2}}-z f(z)=0
$$

The exact solution for the wave function is thus

$$
\Psi(q)==A \operatorname{Ai}(z(q))+B \operatorname{Bi}(z(q)) .
$$

The asymptotic behavior of the Airy functions in the classical region $z \ll-1$, i.e., $a \gg \sqrt{\frac{3}{\Lambda}}$, is

$$
\begin{gathered}
\operatorname{Ai}(\mathrm{z}) \sim \pi^{-1 / 2}|z|^{-1 / 4} \cos \left(\frac{2}{3}|z|^{3 / 2}-\frac{\pi}{4}\right), \\
\operatorname{Bi}(z) \sim-\pi^{-1 / 2}|z|^{-1 / 4} \sin \left(\frac{2}{3}|z|^{3 / 2}-\frac{\pi}{4}\right) .
\end{gathered}
$$

On the other hand in the nonclassical regime $z \gg 1$ (which of course can exist only for a very small cosmological constant $\Lambda \ll 1$ ), one has

$$
\begin{gathered}
\operatorname{Ai}(\mathrm{z}) \sim \frac{1}{2} \pi^{-1 / 2}|z|^{-1 / 4} \exp \left\{-\frac{2}{3} z^{3 / 2}\right\}, \\
\operatorname{Bi}(z) \sim \pi^{-1 / 2}|z|^{-1 / 4} \exp \left\{\frac{2}{3} z^{3 / 2}\right\} .
\end{gathered}
$$

Suppose that we interpret the observed condition of an expanding Universe to mean that $\Psi$ should be an eigenstate of momentum

$$
-i \hbar \frac{d}{d q} \Psi=p \Psi
$$

where by identifying the eigenvalue with the classical momentum, Eq. (30), we have

$$
p=\pi_{q}^{\mathrm{cl}}=-\frac{3}{2 N} \dot{q}<0,
$$

for an expanding Universe. Thus we need

$$
\begin{aligned}
\Psi_{\mathrm{out}} & \sim e^{-i \frac{6}{\hbar \Lambda}\left(\frac{\Lambda q}{3}-1\right)^{3 / 2}+i \frac{\pi}{4}} \\
& \propto \operatorname{Ai}(\mathrm{z}(\mathrm{q}))+\operatorname{iBi}(\mathrm{z}(\mathrm{q})),
\end{aligned}
$$

which gives $p=-3\left(\frac{\Lambda q}{3}-1\right)^{1 / 2}<0$, and hence $\dot{a}>0$. Thus the corresponding under the barrier (i.e., for $\frac{\Lambda q}{3}<1$ ) wave function is

$$
\begin{aligned}
\Psi_{\text {under }}(q)= & A \pi^{-1 / 2}\left[\left(\frac{3}{\hbar^{2} \Lambda^{2}}\right)^{1 / 3}(3-\Lambda q)\right]^{-1 / 4} \\
& \times\left[\frac{1}{2} e^{-\frac{6}{\hbar \Lambda}\left(1-\frac{\Lambda q}{3}\right)^{3 / 2}}+i e^{\frac{6}{\hbar \Lambda}\left(1-\frac{\Lambda q}{3}\right)^{3 / 2}}\right] .
\end{aligned}
$$

This is Vilenkin's tunneling wave function proposal. The condition that the observed Universe is expanding (much as we observe electrons coming out of the nucleus in $\beta$ decay) is used to impose this outgoing boundary condition. Note that we have not normalized these functions. Indeed it is not clear to us whether wave functionals in a quantum field theory (let alone in quantum gravity) are even in principle normalizable. However, the relative probability may still make sense. So the relevant probability for tunneling from nothing is

$$
P_{\text {Tunnel }}(a ; 0) \equiv \frac{\left|\Psi_{\text {out }}(q)\right|^{2}}{\left|\Psi_{\text {under }}(0)\right|^{2}} \sim \exp \left(-\frac{12}{\hbar \Lambda}\right) .
$$

On the other hand Hartle and Hawking argued that the under the barrier wave function is given by Euclidean quantum gravity. This amounts to demanding that the first term in Eq. (43) be the only allowed wave function (i.e., we must pick the solution Ai). Then in the large $q$ regime

$$
\Psi \sim \operatorname{Ai}(z(q)) \rightarrow \cos \left[\frac{6}{\hbar \Lambda}\left(\frac{\Lambda q}{3}-1\right)^{3 / 2}-\frac{\pi}{4}\right],
$$

corresponding to a superposition of an expanding and contracting Universe. In other words one does not have a classical expanding background, and needs to appeal to some sort of decoherence argument to account for the observed expanding Universe. In this case

$$
P_{\mathrm{HH}}(a ; 0) \equiv \frac{\left|\Psi_{\text {out }}(q)\right|^{2}}{\left|\Psi_{\text {under }}(0)\right|^{2}} \sim \exp \left(\frac{12}{\hbar \Lambda}\right) .
$$

All of this is well known and is included here for completeness and to set the stage for identifying the fluctuation spectrum around these solutions.

\section{Fluctuations}

Now one might ask whether the ambiguity as to which wave function is physical might be fixed by the inclusion of tensor perturbations. According to Refs. [10,11], the leading order calculation of tensor perturbations has the wrong (inverse Gaussian) sign for both wave functions, implying that there is no smooth beginning to the Universe in either case. This is contrary to the claim of Ref. [13] (and citations therein) that $\mathrm{HH}$ leads to Gaussian fluctuations, while the tunneling wave function is unstable to quadratic fluctuations. 
The tensor modes are expanded in $S_{3}$ spherical harmonics labeled by integers $l, m, n$. Suppressing the last two indices, the action for a mode $\phi_{l}$ in the de Sitter background is, to quadratic order [11],

$$
S_{l}=\frac{1}{2} \int d t\left[q^{2} \frac{\dot{\phi}_{l}^{2}}{N}-N l(l+2) \phi_{l}^{2}\right] .
$$

So (dropping also the subscript $l$ )

$$
\pi_{\phi}=q^{2} \frac{\dot{\phi}}{N}, \quad \dot{\phi}=\frac{N \pi_{\phi}}{q^{2}},
$$

giving the total Hamiltonian

$$
\mathcal{H}=N H=N\left\{-\frac{1}{3} \pi_{q}^{2}+(\Lambda q-3)+\frac{\pi_{\phi}^{2}}{2 q^{2}}+\frac{1}{2} l(l+2) \phi^{2}\right\} .
$$

The WdW equation is $\left(\pi_{q} \rightarrow-i \hbar \partial / \partial q, \pi_{\phi} \rightarrow-i \hbar \partial / \partial / \partial \phi\right)$

$$
\left\{+\frac{\hbar^{2}}{3} \frac{\partial^{2}}{\partial q^{2}}+(\Lambda q-3)-\frac{\hbar^{2}}{2 q^{2}} \frac{\partial^{2}}{\partial \phi^{2}}+\frac{1}{2} l(l+2) \phi^{2}\right\} \Psi=0 .
$$

Building on the solution in the absence of $\phi$, we try

$$
\Psi[q, \phi] \sim \exp \left\{\frac{1}{\hbar}\left(\frac{6}{\Lambda} c_{1}\left(1-\frac{\Lambda}{3} q\right)^{3 / 2}+c_{2} \frac{1}{2} q \phi^{2} \sigma(q)\right)\right\}
$$

where $c_{1}= \pm 1, c_{2}= \pm 1$, and $\sigma_{l}$ is a function that is to be determined by Eq. (50). Computing derivatives, we get

$$
\begin{aligned}
\partial_{q} \Psi & =\frac{1}{\hbar}\left\{-c_{1} 3\left(1-\frac{\Lambda}{3} q\right)^{1 / 2}+c_{2} \frac{1}{2} \phi^{2}\left(\sigma(q)+q \sigma^{\prime}(q)\right)\right\} \Psi \\
\frac{\hbar^{2}}{3} \partial_{q}^{2} \Psi & =\left\{(3-\Lambda q)-c_{1} c_{2} \phi^{2}\left(\sigma(q)+q \sigma^{\prime}(q)\right)\left(1-\frac{\Lambda}{3} q\right)^{1 / 2}+O\left(\phi^{4}\right)+O(\hbar)\right\} \Psi, \\
-\frac{\hbar^{2}}{2 q^{2}} \partial_{\phi}^{2} \Psi & =\left[-\frac{1}{2} \phi^{2} \sigma^{2}(q)+O(\hbar)\right] \Psi .
\end{aligned}
$$

Thus ignoring the $\phi^{4}$ and $\hbar$ corrections, the WdW equation (50) becomes

$$
\left[-c_{1} c_{2} \phi^{2}\left(\sigma(q)+q \sigma^{\prime}(q)\right)\left(1-\frac{\Lambda}{3} q\right)^{1 / 2}-\frac{1}{2} \phi^{2} \sigma^{2}(q)+\frac{1}{2} l(l+2) \phi^{2}\right] \Psi=0 .
$$

Thus $\sigma_{l}$ is required to be a solution of

$$
-c_{1} c_{2}\left(\sigma(q)+q \sigma^{\prime}(q)\right)\left(1-\frac{\Lambda}{3} q\right)^{1 / 2}-\frac{1}{2} \sigma^{2}(q)+\frac{1}{2} l(l+2)=0 .
$$

Putting

$$
\sigma(q)=\frac{g_{l}}{\left(1-\frac{\Lambda}{3} q\right)^{1 / 2}+f_{l}}
$$

we see that Eq. (54) is satisfied with

$$
f_{l}= \pm(l+1), \quad g_{l}=c_{1} c_{2} l(l+2) .
$$

Thus (since $c_{2}^{2}=1$ ) we have the four solutions for the wave function [up to terms $O\left(\phi^{4}\right), O(\hbar)$ in the exponent]:

$$
\begin{aligned}
\Psi & \sim \exp \left\{\frac{c_{1}}{\hbar}\left(\frac{6}{\Lambda}\left(1-\frac{\Lambda}{3} q\right)^{3 / 2}+\frac{1}{2} q \phi^{2} \frac{l(l+2)}{\left(1-\frac{\Lambda}{3} q\right)^{1 / 2} \pm(l+1)}\right)\right\} \\
& =\exp \left\{\frac{c_{1}}{\hbar}\left(\frac{6}{\Lambda}\left(1-\frac{\Lambda}{3} q\right)^{3 / 2}+\frac{1}{2} q \phi^{2} \frac{l(l+2)\left(-\left(1-\frac{\Lambda}{3} q\right)^{1 / 2} \pm(l+1)\right)}{\frac{\Lambda}{3} q+l(l+2)}\right)\right\} .
\end{aligned}
$$


Similarly we have four possible solutions in the classically allowed region,

$$
\begin{aligned}
\Psi & \sim \exp \left\{\frac{c_{1}}{\hbar}\left(\frac{6}{\Lambda} i\left(\frac{\Lambda}{3} q-1\right)^{3 / 2}-\frac{1}{2} q \phi^{2} \frac{l(l+2)}{i\left(\frac{\Lambda}{3} q-1\right)^{1 / 2} \pm(l+1)}\right)\right\} \\
& =\exp \left\{\frac{c_{1}}{\hbar}\left(\frac{6}{\Lambda} i\left(\frac{\Lambda}{3} q-1\right)^{3 / 2}+\frac{1}{2} q \phi^{2} \frac{l(l+2)\left(i\left(\frac{\Lambda}{3} q-1\right)^{1 / 2} \mp(l+1)\right)}{\frac{\Lambda}{3} q+l(l+2)}\right)\right\}
\end{aligned}
$$

Note that these four solutions are in agreement with the solutions for the classical action including quadratic fluctuations given in Eq. (30) of Ref. [11]. It is clear that two of the four solutions (both under and outside the barrier) give Gaussian fluctuations, while two give inverse Gaussian fluctuations. Obviously the general solution which would be a linear superposition of these four solutions will necessarily have components which would invalidate the smooth background which is the starting assumption of this analysis. Feldbrugge et al. [11] claim that their path integral argument (essentially involving an ordinary integral over the lapse $N$ ) necessarily includes a non-Gaussian component in both the $\mathrm{T}$ and $\mathrm{HH}$ solutions. In other words depending on the choice of contour (i.e., with a given sign for $c_{1}$ ), one needs to include both \pm signs [corresponding to the two signs for $f_{l}$ in Eq. (55)] in the solution.

Nevertheless as we will discuss below, once one uses the matching conditions to evaluate the wave function in the classical regime, it will be seen that we get different linear combinations of the under barrier wave functions. In fact one can choose to pick those linear combinations which admit only Gaussian fluctuations [with the appropriate choice of sign in $f_{l}$ in Eq. (55)]. This can be done for both the tunneling wave function and the Hartle-Hawking wave function.

Before we discuss this, we would like to point out that the wave function necessarily contains non-Gaussian fluctuations. Even ignoring $\hbar$ corrections, there are $O\left(\phi^{4}\right)$ terms

$$
\frac{1}{12}\left(\sigma(q)+q \sigma^{\prime}(q)\right)^{2} \phi^{4}
$$

in Eq. (52) which imply that the quadratic (in $\phi$ ) term in the $\log$ of the wave function $\Psi$ in Eq. (51) needs to include a quartic term in $\phi$. This does not necessarily imply measurable non-Gaussianities in the cosmic microwave background spectrum since it is possible that they are significant only at points in field space where the perturbative analysis of this paper-namely, the expansion in fluctuations $\phi$ - breaks down. $^{3}$

\section{Wave function with fluctuations}

Using the matching conditions for the zeroth order (in fluctuations) wave functions given by the asymptotic behavior of the Airy functions (38)-(41), and the correlation that we found between the sign of the fluctuations and the particular solution to the zeroth order equation (56) [see also Eqs. (72) and (74)], we can now write down the wave function in the classically forbidden and allowed regions for the asymptotic regimes and for small $\phi$ fluctuations. To simplify the formulas, let us first make the following definitions:

$$
\lambda(q) \equiv \frac{6}{\Lambda}\left|\left(1-\frac{\Lambda q}{3}\right)\right|^{3 / 2}, \quad \Delta \equiv \frac{\Lambda}{3} q+l(l+2)>0 .
$$

For $\frac{\Lambda q}{3} \ll 1$ we have

$$
\begin{aligned}
& \left.\Psi_{\text {in }}(q, \phi)=A_{+} e^{-\frac{1}{\hbar}\left\{\lambda(q)+\frac{1}{2} q \phi^{2} \frac{\left(l(l+2)\left(-\left(1-\frac{1}{3} q\right)^{1 / 2}+(l+1)\right)\right.}{\Delta}\right.}\right\} \\
& +A_{-} e^{-\frac{1}{\hbar}\left\{\lambda(q)+\frac{1}{2} q \phi^{2} \frac{\left(l(l+2)\left(-\left(1-\frac{A}{3} q\right)^{1 / 2}-(l+1)\right)\right.}{\Delta}\right\}} \\
& \left.+B_{+} e^{+\frac{1}{\hbar}\left\{\lambda(q)+\frac{1}{2} q \phi^{2} \frac{l(l+2)\left(-\left(1-\frac{1}{3} q\right)^{1 / 2}+(l+1)\right)}{\Delta}\right.}\right\} \\
& \left.+B_{-} e^{+\frac{1}{\hbar}\left\{\lambda(q)+\frac{1}{2} q \phi^{2} \frac{2(l+2)\left(-\left(1-\frac{A}{3} q\right)^{1 / 2}-(l+1)\right)}{\Delta}\right.}\right\} \text {. }
\end{aligned}
$$

Note that the $A_{+}$and $B_{-}$terms have Gaussian fluctuations while the $A_{-}$and $B_{+}$terms have inverse Gaussian fluctuations. Hence one might choose the solution with

$$
A_{-}=B_{+}=0
$$

in order to avoid quadratic instabilities. Of course this is not a guarantee that the solutions are indeed stable since we have nothing to say about the sign of higher order fluctuations.

In the asymptotic classical regime $\Lambda q / 3 \gg 1$ the general solution may be written as (we include a constant phase factor for later convenience)

$$
\begin{aligned}
\Psi_{\text {out }}(q, \phi)= & C_{+}^{-} e^{\frac{1}{\hbar}\left\{i \lambda(q)-\frac{q}{2} \phi^{2} \frac{l^{(l(l+2)}}{D_{+}}\right\}-i_{4}^{\frac{\pi}{4}}}+C_{-}^{+} e^{-\frac{1}{\hbar}\left\{i \lambda(q)-\frac{q}{2} \phi^{2} \frac{2^{(l(l+2)}}{D_{+}}\right\}+i_{4}^{\frac{\pi}{4}}} \\
& +C_{+}^{+} e^{\frac{1}{\hbar}\left\{i \lambda(q)-\frac{q}{2} \phi^{2} \frac{2(l+2)}{D_{-}}\right\}-i_{4}^{\frac{\pi}{4}}}+C_{-}^{-} e^{-\frac{1}{\hbar}\left\{i \lambda(q)-\frac{q}{2} \phi^{2} \frac{2(l+2)}{D_{-}}\right\}+i_{4}^{\frac{\pi}{4}}}
\end{aligned}
$$

with

\footnotetext{
${ }^{3}$ I thank an anonymous referee for emphasizing this.
} 


$$
\frac{1}{D_{ \pm}}=\frac{-i(\Lambda q / 3-1)^{1 / 2} \pm(l+1)}{\Delta}
$$

In Eq. (61) as well as in Eq. (59) the particular combination of the fluctuation term $\propto \phi^{2}$ and the background term $\propto \lambda(q)$ is determined by the Wheeler-DeWitt equation, as indicated in Eq. (58). To avoid having solutions which are unstable to quadratic fluctuations in the classical region, we need to set

$$
C_{-}^{+}=0, \quad C_{+}^{+}=0
$$

Let us now impose the requirement of suppressed quadratic fluctuations in both the inside and outside wave functions, and then match the two in the limit where $\phi=0$. In this case we may use the matching conditions of the unperturbed theory (38)-(41), which imply

$$
\begin{aligned}
\frac{1}{2} e^{-\frac{\lambda(q)}{\hbar}} & \longleftrightarrow \frac{1}{2}\left\{e^{\frac{i}{\hbar} \lambda(q)-i \frac{\pi}{4}}+e^{-\frac{i}{\hbar} \lambda(q)+i \frac{\pi}{4}}\right\}, \\
e^{\frac{\lambda(q)}{\hbar}} & \longleftrightarrow \frac{1}{2 i}\left\{e^{\frac{i}{\hbar} \lambda(q)-i \frac{\pi}{4}}-e^{-\frac{i}{\hbar} \lambda(q)+i \frac{\pi}{4}}\right\},
\end{aligned}
$$

where the lhs of each relation corresponds to a classically forbidden region, and the rhs to a classically allowed region solution. These conditions then determine $C_{+}^{-}=A_{+}-\frac{1}{2 i} B_{-}$, $C_{-}^{-}=A_{+}+\frac{1}{2 i} B_{-}$. For the sake of clarity let us then write out explicitly the solutions with suppressed Gaussians in the two regions ${ }^{4}$ :

$$
\begin{aligned}
\Psi_{\text {in }}(q, \phi)= & A_{+} e^{-\frac{1}{\hbar}\left\{\lambda(q)+\frac{1}{2} q \phi^{2} \frac{l(l+2)\left(-\left(1-\frac{\Lambda}{3} q\right)^{1 / 2}+(l+1)\right)}{\Delta}\right\}} \\
& +B_{-} e^{+\frac{1}{\hbar}\left\{\lambda(q)+\frac{1}{2} q \phi^{2} \frac{l(l+2)\left(-\left(1-\frac{\Lambda}{3} q\right)^{1 / 2}-(l+1)\right)}{\Delta}\right\}} \\
\Psi_{\text {out }}(q, \phi)= & \left(A_{+}-\frac{1}{2 i} B_{-}\right) e^{\frac{1}{\hbar}\left\{i \lambda(q)-\frac{q}{2} \phi^{2} \frac{l(l+2)}{D_{+}}\right\}-i \frac{\pi}{4}} \\
& +\left(A_{+}+\frac{1}{2 i} B_{-}\right) e^{-\frac{1}{\hbar}\left\{i \lambda(q)-\frac{q}{2} \phi^{2} \frac{l(l+2)}{D_{-}}\right\}+i \frac{\pi}{4}} .
\end{aligned}
$$

Consider now Vilenkin's tunneling wave function case. The boundary condition here is that there is only an outgoing component in the classical region corresponding to an expanding Universe, which means setting $A_{+}+B_{-} / 2 i=0$. Thus we get

$$
\Psi_{\mathrm{out}}^{(T)}(q, \phi)=2 A_{+} e^{-\frac{1}{\hbar}\left\{i \lambda(q)-\frac{q}{2} \phi^{2} \frac{l(l+2)}{D_{-}}\right\}+i \frac{\pi}{4}}
$$

This should be compared with $\Psi_{\text {in }}$,

\footnotetext{
${ }^{4} \mathrm{~A}$ similar analysis for the case of tunneling boundary conditions was done more than 30 years ago by Vachaspati and Vilenkin [18].
}

$$
\Psi_{\text {in }}^{(T)}(q \rightarrow 0)=A_{+}\left[e^{-\frac{6}{\hbar \Lambda}}-2 i e^{\frac{6}{\hbar \Lambda}}\right] .
$$

The probability of the Universe emerging in a BunchDavies vacuum relative to remaining in a state of nothing (i.e., with zero sale factor $a=\sqrt{q}=0$ ) is (after restoring the $2 \pi^{2}$ factor which we had dropped)

$P^{(T)}(q, \phi)=\frac{\left|\Psi_{\mathrm{out}}^{(T)}(q, \phi)\right|^{2}}{\left|\Psi_{\mathrm{in}}^{(T)}(q \rightarrow 0)\right|^{2}}=e^{-24 \pi^{2} / \hbar \Lambda} e^{-\frac{2 \pi^{2}}{\hbar} q \phi^{2} \frac{l(l+1)(l+2)}{\Delta}}$.

Now let us consider the Hartle-Hawking case. Here if we insist that the boundary conditions are given by Euclidean quantum gravity, we would need the wave function to be real. In this case using Eq. (62) in Eq. (61) and imposing reality, we get (we choose $\Im A_{+}=0$ so $\Re B_{-}=0$ )

$$
\begin{aligned}
\Psi_{\mathrm{out}}^{(\mathrm{HH})}= & 4 A_{+} \cos \left[\frac{1}{\hbar}(\lambda(q)\right. \\
& \left.\left.+\frac{q \phi^{2}}{2}(\Lambda q / 3-1)^{1 / 2} \frac{l(l+2)}{\Delta}\right)-\frac{\pi}{4}\right] e^{-\frac{2 \pi^{2}}{\hbar} \phi^{2} \frac{(l(l+1)(l+2)}{2 \Delta}},
\end{aligned}
$$

in agreement with Eq. 3.23 of Ref. [13]. Furthermore the under the barrier solution in this case in the limit of zero scale factor is

$$
\Psi_{\text {in }}^{(\mathrm{HH})}(q \rightarrow 0)=2 A_{+} e^{-\frac{6}{\hbar \Lambda}} .
$$

Thus we have (after restoring the factor of $2 \pi^{2}$ )

$$
\begin{aligned}
P^{(\mathrm{HH})}(q, \phi)= & \frac{\left|\Psi_{\mathrm{out}}^{(\mathrm{HH})}(q, \phi)\right|^{2}}{\left|\Psi_{\mathrm{in}}^{(\mathrm{HH})}(q \rightarrow 0)\right|^{2}} \\
= & e^{24 \pi^{2} / \hbar \Lambda} e^{-\frac{2 \pi^{2}}{\hbar} q \phi^{2} \frac{(l(l+1)(l+2)}{\Delta}} \\
& \times 4 \cos ^{2}\left[\frac{2 \pi^{2}}{\hbar}(\lambda(q)\right. \\
& \left.\left.+\frac{q \phi^{2}}{2}(\Lambda q / 3-1)^{1 / 2} \frac{l(l+2)}{\Delta}\right)-\frac{\pi}{4}\right] .
\end{aligned}
$$

The Hartle-Hawking wave function is time symmetric between an expanding and a contracting Universe. The observed Universe is of course expanding, so somehow the two branches must decohere-in which case the only difference between the two probabilities is in the prefactorwith the tunneling case (as is well known) favoring a larger cosmological constant, and the Hartle-Hawking case favoring a smaller CC. 


\section{E. Solving the Hamilton-Jacobi equation beyond quadratic order}

In the previous subsection we considered just the quadratic fluctuations around the minisuperspace $\mathrm{HH}$ and tunneling solutions. However, as we mentioned at the end of Sec. III C, it is clear that the wave function necessarily contains non-Gaussianities.

To investigate the solutions systematically, it is convenient to write

$$
\Psi[q, \phi]=e^{\frac{i}{h} S[q, \phi]} .
$$

Substituting in the WdW equation (50), we have the Hamilton-Jacobi (HJ) equation plus its quantum correction:

$$
\begin{aligned}
- & \frac{1}{3}\left(\frac{\partial S}{\partial q}\right)^{2}+(\Lambda q-3)+\frac{1}{2 q^{2}}\left(\frac{\partial S}{\partial \phi}\right)^{2}+\frac{1}{2} l(l+2) \phi^{2} \\
& +i \hbar\left(\frac{1}{3} \frac{\partial^{2} S}{\partial q^{2}}-\frac{1}{2 q^{2}} \frac{\partial^{2} S}{\partial \phi^{2}}\right)=0
\end{aligned}
$$

The limit $\hbar \rightarrow 0$ give the classical Hamilton-Jacobi equation. Ignoring the quantum correction, we try a solution of the form (as before, an overall factor of $2 \pi^{2}$ is understood)

$$
i S[q, \phi]=c_{1}\left[\frac{6}{\Lambda}\left(1-\frac{\Lambda}{3} q\right)^{3 / 2}+q\left(\sum_{n=1}^{\infty} \frac{1}{2 n !} \sigma_{n}(q) \phi^{2 n}\right)\right] .
$$

The HJ equation then gives

$$
\begin{aligned}
- & 2\left(1-\frac{\Lambda}{3} q\right)^{1 / 2} \sum_{n=1}^{\infty} \frac{1}{2 n !}\left(\sigma_{n}(q)+q \sigma_{n}^{\prime}(q)\right) \phi^{2 n} \\
& +\left(\sum_{n=1}^{\infty} \frac{1}{2 n !}\left(\sigma_{n}(q)+q \sigma_{n}^{\prime}(q)\right) \phi^{2 n}\right)^{2} \\
& -\frac{1}{2}\left(\sum_{n=1}^{\infty} \frac{1}{(2 n-1) !} \sigma_{n}(q) \phi^{2 n-1}\right)^{2}+\frac{1}{2} l(l+2) \phi^{2}=0 .
\end{aligned}
$$

Equating powers of $\phi^{2}$ gives a set of recursion relations which in principle can be solved iteratively to determine $\sigma_{n}$. For instance from the coefficient of $\phi^{2}$ we get [see Eqs. (54) and (55)]

$$
-\left(1-\frac{\Lambda}{3} q\right)^{1 / 2}\left(\sigma_{1}(q)+q \sigma_{1}^{\prime}(q)\right)-\frac{1}{2} \sigma_{1}^{2}+\frac{1}{2} l(l+2)=0
$$

which is solved by

$$
\sigma_{1}=\frac{l(l+2)}{\left(1-\frac{\Lambda}{3} q\right)^{1 / 2} \pm(l+1)} .
$$

From the coefficient of $\phi^{4}$ we also get

$$
\frac{2}{4 !}\left(1-\frac{\Lambda}{3} q\right)^{1 / 2}\left(\sigma_{2}+q \sigma_{2}^{\prime}\right)+\frac{1}{6} \sigma_{1} \sigma_{2}=\frac{1}{4}\left(\sigma_{1}+q \sigma_{1}^{\prime}\right)^{2} .
$$

Since $\sigma_{1}+q \sigma_{1}^{\prime} \neq 0$, this shows that $\sigma_{2} \neq 0$. Clearly the higher order terms will all be nonzero. Note also that in the classical regime $\left(\frac{\Lambda}{3} q>1, a>a_{d S} \equiv \sqrt{\frac{3}{\Lambda}}\right)$ the sigmas are complex since, as we remarked before,

$$
\sigma_{1}=\frac{l(l+2)\left[ \pm(l+1)-i \sqrt{\frac{\Lambda}{3} q-1}\right]}{\frac{\Lambda}{3} q-1+(l+1)^{2}}, \text { etc. }
$$

The above calculation seems to indicate that the solution of the WdW equation in the minisuperspace approximation has non-Gaussian terms. It is however unclear whether all of them are suppressed under the same conditions that enabled us to get suppressed Gaussian fluctuations.

In view of the importance of this question, let us investigate this further using a slightly different parametrizationi.e., the one used by Vachaspati and Vilenkin [18]. First we rewrite the WdW equation (50) in terms of the scale factor $a$ to get

$$
\begin{array}{r}
\left\{+\hbar^{2}\left(\frac{\partial^{2}}{\partial a^{2}}+p \frac{\partial}{\partial a}\right)+12 a^{4}\left(\Lambda a^{2}-3\right)\right. \\
\left.-6 \hbar^{2} \frac{\partial^{2}}{\partial \phi^{2}}+6 l(l+2) a^{4} \phi^{2}\right\} \Psi=0 .
\end{array}
$$

This is essentially (apart from a trivial normalization difference) the equation analyzed in Ref. [18] (but for a given mode $\phi \equiv \phi_{l}$ ) except that the direct substitution $q=a^{2}$ would have given us $p=-1$ in the above, whereas they chose $p=+1$. But this is just a difference in factor ordering which is relevant only for the $O(\hbar)$ correction, which we ignore. So let us work with the latter choice for $p$. In this case we may substitute $x=\ln a$ to get

$$
\begin{array}{r}
\left\{+\hbar^{2} \frac{\partial^{2}}{\partial x^{2}}+-6 \hbar^{2} \frac{\partial^{2}}{\partial \phi^{2}}-V(x, \phi)\right\} \Psi=0, \\
V(x, \phi)=V_{0}(x)+V_{2}(x) \phi^{2} \\
\equiv-12 e^{4 x}\left(\Lambda e^{2 x}-3\right)-6 l(l+2) e^{4 x} \phi^{2} .
\end{array}
$$

We write the wave function as (we will write out explicitly only the under the barrier growing mode)

$\Psi(x, \phi)=\exp \left[-S_{0}(x)-\frac{1}{2} S_{2}(x) \phi^{2}-\frac{1}{4} S_{4}(x) \phi^{4}+O\left(\phi^{6}\right)\right]$.

Substituting in Eq. (76), we get a set of recursion relations as before-namely (denoting by a prime derivation with respect to $x$ ), 


$$
\begin{gathered}
S_{0}^{\prime 2}=V_{0} \\
S_{0}^{\prime} S_{2}^{\prime}-S_{2}^{2}=V_{2} \\
\frac{1}{2} S_{0}^{\prime} S_{4}^{\prime}+\frac{1}{4}\left(S_{2}^{\prime}\right)^{2}-2 S_{2} S_{4}=0 \\
\frac{1}{t} S_{0}^{\prime} S_{2 t}^{\prime}+\sum_{r=1}^{t-1} \frac{S_{2 r}^{\prime}}{2 r} \frac{S_{2(t-r)}^{\prime}}{2(t-r)}-6 \sum_{r=1}^{t} S_{2 r} S_{2(t+1-r)}=0 \text { for } t \geq 2 .
\end{gathered}
$$

Clearly all of the coefficients $S_{2 t}$ may be recursively determined in principle. Solving the equation for $S_{4}$ for instance, we get

$S_{4}(x)=-\frac{1}{2} e^{24 \int_{-\infty}^{x} d x^{\prime} S_{0}^{S_{2}}\left(x^{\prime}\right)} \int_{-\infty}^{x} d x^{\prime} e^{-4 \int_{-\infty}^{x^{\prime}} d x^{\prime \prime} \frac{S_{2}}{S_{0}^{\prime}}\left(x^{\prime \prime}\right)} \frac{\left(S_{2}^{\prime}\left(x^{\prime}\right)\right)^{2}}{S_{0}^{\prime}\left(x^{\prime}\right)}$.

Once a choice of $S_{0}^{\prime}= \pm \sqrt{V_{0}}$ is made, this equation [together with the choice of solution for Eq. (80), which is essentially given by $q \sigma_{1}$ - see Eqs. (72) and (74)] determines uniquely the coefficient $S_{4}$. In fact in the classically forbidden region where $V_{0}>0$ and $S_{2}^{\prime}$ is real, we see that for the choice $S_{0}^{\prime}=-\sqrt{V_{0}}$ [giving $S_{0}=+2 \sqrt{3}\left(3-\Lambda a^{2}\right)^{3 / 2}$ ], we have $S_{4}>0$ corresponding to suppressed quartic fluctuations in the rising under the barrier wave function, but the choice $S_{0}^{\prime}=+\sqrt{V_{0}}$ gives growing quartic fluctuations signaling an instability in the falling under the barrier wave function.

What does this imply for the tunneling vs the HartleHawking wave function? For the latter case (since we can set $B_{-}=0$ ) we have suppressed quartic fluctuations. However, for the former case since $B_{-}$is necessarily nonzero, we would have unsuppressed quartic fluctuations in the under the barrier wave function. If this sign persists for all higher order (i.e., $\phi^{2 t}$ ) terms, then clearly the requirement of suppressed fluctuations will favor the $\mathrm{HH}$ wave function. Unfortunately [as can be seen from Eq. (82)] analyzing the higher order (i.e., $2 t \geq 6$ ) fluctuations is not straightforward, so at this point we cannot say anything about these, and a definite conclusion regarding these alternative solutions to the problem of tunneling from nothing cannot be made.

While we cannot say anything about the sign of the higher order (i.e., beyond quartic order) fluctuations, the recursion relations (73), (81), and (83) (barring some unlikely cancellations) imply that this framework may in general lead to non-Gaussianities. However, as we observed earlier, this does not necessarily imply a breakdown of the Bunch-Davies vacuum ansatz since it may be the case that these quartic (and higher order) corrections become significant only at values of $\phi$ where our expansion breaks down.

\section{CONCLUSIONS}

We have argued that there is no particular advantage to the saddle point (Picard-Lefschetz) method in solving the WdW equation in the minisuperspace truncation. Contrary to the work of Feldbrugge et al. [9-12], which ascribes certain a priori criteria on properties of the integral over the lapse and furthermore essentially computes a propagator rather than a wave function, our point of view is essentially similar to that of Diaz and co-workers $[13,14]$, where the functional integral is treated as a means towards computing solutions to the WdW equation.

We have also argued that whether or not one picks the Hartle-Hawking or the tunneling wave function is a matter of choosing one or the other boundary condition, so it must be determined by some physical input and is not a matter of consistency of the saddle point method. Furthermore we have shown how to include fluctuations around the minisuperspace model, and indeed the general solution does contain terms with inverse Gaussian fluctuations, as shown by Feldbrugge et al. [10,11]. However, we have demonstrated that one can choose to set some of the arbitrary constants by multiplying such terms by zero, so properly interpreted, the quadratic fluctuations around both competing wave functions are indeed of Gaussian form and are suppressed.

On the other hand we have been unable to say anything definitive about the sign of higher order fluctuations (apart from the quartic one, which appears to have the wrong sign for the tunneling wave function), hence it is difficult to rule out either formulation of quantum cosmology based on purely theoretical grounds (as was the claim of Refs. [10,11]). However, as argued at the end of the previous section, even if the stability issue was settled it is hard to see how non-Gaussianities could be suppressed (as required observationally) in this framework.

\section{ACKNOWLEDGMENTS}

I wish to thank Cliff Burgess and Fernando Quevedo for the discussions and comments on the manuscript, and Alex Vilenkin for a question on a previous version of this paper, which led me discuss the general solution in detail. I also wish to acknowledge the hospitality of the Abdus Salam ICTP, where part of this work was done, and the receipt of partial support from the Dean of the College of Arts and Sciences at the University of Colorado, Boulder. 
[1] A. Borde, A. H. Guth, and A. Vilenkin, Phys. Rev. Lett. 90, 151301 (2003).

[2] J. M. Maldacena, J. High Energy Phys. 05 (2003) 013.

[3] S. Weinberg, Phys. Rev. D 72, 043514 (2005).

[4] J. B. Hartle and S. W. Hawking, Phys. Rev. D 28, 2960 (1983).

[5] A. Vilenkin, Phys. Lett. 117B, 25 (1982).

[6] A. Vilenkin and M. Yamada, Phys. Rev. D 98, 066003 (2018).

[7] A. Yu. Kamenshchik, A. Tronconi, T. Vardanyan, and G. Venturi, Int. J. Mod. Phys. D 28, 1950073 (2019).

[8] M. Bojowald and S. Brahma, Phys. Rev. Lett. 121, 201301 (2018).

[9] J. Feldbrugge, J.-L. Lehners, and N. Turok, Phys. Rev. D 95, 103508 (2017).

[10] J. Feldbrugge, J.-L. Lehners, and N. Turok, Phys. Rev. Lett. 119, 171301 (2017).
[11] J. Feldbrugge, J.-L. Lehners, and N. Turok, Phys. Rev. D 97, 023509 (2018).

[12] J. Feldbrugge, J.-L. Lehners, and N. Turok, Universe 4, 100 (2018).

[13] J. Diaz Dorronsoro, J. J. Halliwell, J. B. Hartle, T. Hertog, and O. Janssen, Phys. Rev. D 96, 043505 (2017).

[14] J. Diaz Dorronsoro, J. J. Halliwell, J. B. Hartle, T. Hertog, O. Janssen, and Y. Vreys, Phys. Rev. Lett. 121, 081302 (2018).

[15] J. J. Halliwell, J. B. Hartle, and T. Hertog, Phys. Rev. D 99, 043526 (2019).

[16] J. D. Brown and C. Teitelboim, Nucl. Phys. B297, 787 (1988).

[17] E. Merzbacher, Quantum Mechanics, 3rd ed. (Wiley, New York, 1998).

[18] T. Vachaspati and A. Vilenkin, Phys. Rev. D 37, 898 (1988). 